\title{
Isomorphism on Irregular Intuitionistic Fuzzy Graphs and Its Complements
}

\author{
${ }^{1}$ S. Yahya Mohamed and ${ }^{2}$ R.JahirHussain \\ ${ }^{\prime}$ P.G Department of Mathematics, Govt. Arts College, Tiruchirappalli-620 022, India \\ ${ }^{2} P$.G and Research Department of Mathematics, Jamal Mohamed College, Tiruchirappalli-620 020, India
}

\begin{abstract}
In this paper, We study more results of Isomorphism on highly Irregular intuitionistic fuzzy graphs discussed. Isomorphism on neighbourly Irregular intuitionistic fuzzy graphs and its complement are established. Also we extend the isomorphic properties of Neighbourly total Irregular intuitionistic fuzzy graphs.
\end{abstract}

Keywords:Intuitionistic fuzzy graph, degree, total degree, neigbourly IFG, neighbourly Total IFG.

\section{Introduction:}

K.R. Bhutani in [5] introduced the concept of weak isomorphism and isomorphism between fuzzy graphs. A. NagoorGani and J. Malarvizhi in[6] proved that Isomorphism between fuzzy graphs is an equivalence relations and they have discussed some properties of complementary fuzzy graphs. Atanassov [1] introduced the concept of intuitionistic fuzzy (IF) relations and intuitionistic fuzzy graphs (IFGs). Research on the theory of intuitionistic fuzzy sets (IFSs) has been witnessing an exponential growth in Mathematics and its applications. This ranges from traditional Mathematics to Information Sciences. This leads to consider IFGs and their applications. R. Parvathy and M.G.Karunambigai's paper [2] introduced the concept of IFG and analyzed its components.NagoorGani, A and Sajitha Begum, S [4] defined degree, Order and Size in intuitionistic fuzzy graphs and extend the properties. A.NagoorGaniand R. Latha[3] introduced Irregular fuzzy graphs and discussed some of its properties.A. NagoorGani, et. al[10] defined types of Irregular Intuitionistic fuzzy graphs and discussed some properties. Also R. JahirHussain and S. Yahya Mohamed [11] established properties on Highly irregular Intuitionistic fuzzy graphs and its complements.

In this paper, we discuss more results on isomorphism on highly Irregular intuitionistic fuzzy graphs. Isomorphism on neighbourly Irregular intuitionistic fuzzy graphs and its complements established. Also we extend some result on isomorphism on neighbourlytotal Irregular intuitionistic fuzzy graphs and this concept is useful in Information analysis and computer networks.

\section{Preliminaries}

Definition 2.1: An Intuitionistic fuzzy graph is of the form $\mathrm{G}=(\mathrm{V}, \mathrm{E})$ where

(i) $\mathrm{V}=\left\{\mathrm{v}_{1}, \mathrm{v}_{2}, \ldots, \mathrm{v}_{\mathrm{n}}\right\}$ such that $\mu_{1}: \mathrm{V} \rightarrow[0,1]$ and $\gamma_{1}: \mathrm{V} \rightarrow[0,1]$ denote the degree of membership and nonmembership of the element vi $\epsilon \mathrm{V}$, respectively, and $0 \leq \mu_{1}(\mathrm{vi})+\gamma_{1}(\mathrm{vi}) \leq 1$ for every vi $\epsilon \mathrm{V},(\mathrm{i}=1,2, \ldots \ldots \mathrm{n})$,

(ii) $\mathrm{E} \subseteq \mathrm{Vx} \mathrm{V}$ where $\mu_{2}: \mathrm{VxV} \rightarrow[0,1]$ and $\gamma_{2}: \mathrm{VxV} \rightarrow[0,1]$ are such that $\mu_{2}(\mathrm{vi}, \mathrm{vj}) \leq \min \left[\mu_{1}(\mathrm{vi}), \mu_{1}(\mathrm{vj})\right]$ and $\gamma_{2}(\mathrm{vi}, \mathrm{vj}) \leq \max \left[\gamma_{1}(\mathrm{vi}), \gamma_{1}(\mathrm{vj})\right]$ and $0 \leq \mu_{2}(\mathrm{vi}, \mathrm{vj})+\gamma_{2}(\mathrm{vi}, \mathrm{vj}) \leq 1$ for every $(\mathrm{vi}, \mathrm{vj}) \epsilon \mathrm{E},(\mathrm{i}, \mathrm{j}=1,2, \ldots \ldots \mathrm{n})$

Definition 2.2:Let $G=(V, E)$ be an IFG. Then the degree of a vertex $v$ is defined by $d(v)=(d \mu(v), d \gamma(v))$ where $\mathrm{d} \mu(\mathrm{v})=\Sigma \mathrm{u} \neq \mathrm{v} \mu_{2}(\mathrm{v}, \mathrm{u})$ and $\mathrm{d} \gamma(\mathrm{v})=\Sigma \mathrm{u} \neq \mathrm{v} \gamma_{2}(\mathrm{v}, \mathrm{u})$.

Definition 2.3: The minimum degree of $\mathrm{G}$ is $\delta(\mathrm{G})=(\delta \mu(\mathrm{G}), \delta \gamma(\mathrm{G}))$ where $\delta \mu(\mathrm{G})=\Lambda\{\mathrm{d} \mu(\mathrm{v}) / \mathrm{v} \in \mathrm{V}\}$ and $\delta \gamma(\mathrm{G})=\Lambda\{\mathrm{d} \gamma(\mathrm{v}) / \mathrm{v} \in \mathrm{V}\}$

Definition 2.4: The maximum degree of $\mathrm{G}$ is $\Delta(\mathrm{G})=(\Delta \mu(\mathrm{G}), \Delta \gamma(\mathrm{G}))$ where $\Delta \mu(\mathrm{G})=\mathrm{V}\{\mathrm{d} \mu(\mathrm{v}) / \mathrm{v} \in \mathrm{V}\}$ and $\Delta \gamma(\mathrm{G})=\mathrm{V}\{\mathrm{d} \gamma(\mathrm{v}) / \mathrm{v} \epsilon \mathrm{V}\}$

Definition 2.5: The total degree of a vertex ' $v$ ' is defined as $t(v)=\left(t_{\mu}(v), t_{\gamma}(v)\right)$, where $\mathrm{t}_{\mu}(\mathrm{v})=\Sigma \mathrm{u} \neq \mathrm{v} \mu_{2}(\mathrm{v}, \mathrm{u})+\mu_{1}(\mathrm{v})$ and $\mathrm{t}_{\gamma}(\mathrm{v})=\Sigma \mathrm{u} \neq \mathrm{v} \gamma_{2}(\mathrm{v}, \mathrm{u})+\gamma_{1}(\mathrm{v})$

Definition 2.6: The complement of an IFG G $=(\mathrm{V}, \mathrm{E})$ is denoted by $\bar{G}=(\bar{V}, \bar{E})$ and is defined as i) $\bar{\mu}_{1}(v)=\mu_{1}(v)$ and $\bar{\gamma}_{1}(v)=\gamma_{1}(v)$

ii) $\bar{\mu}_{2}(\mathrm{u}, \mathrm{v})=\mu_{1}(\mathrm{u}) \Lambda \mu_{1}(\mathrm{v})-\mu_{2}(\mathrm{u}, \mathrm{v})$ and $\bar{\gamma}_{2}(\mathrm{u}, \mathrm{v})=\gamma_{1}(\mathrm{u}) \mathrm{V} \gamma_{1}(\mathrm{v})-\gamma_{2}(\mathrm{u}, \mathrm{v})$ for $\mathrm{u}, \mathrm{v}$ in $\mathrm{V}$

Definition 2.7: An Intuitionistic fuzzy graph $G=(V, E)$ is said to be regular, if every vertex has same degree.

Definition 2.8: Let $G=(V, E)$ be IFG. Then $G$ is irregular, if there is a vertex which is adjacent to vertices with distinct degrees. 
Isomorphism on Irregular Intuitionistic Fuzzy Graphs And Its Complements

\section{Definition2.9:}

A homomorphism of Intuitionistic fuzzy graphs $h: G \rightarrow G^{\prime}$ is a map $h: S \rightarrow S^{\prime}$ which satisfies

(i) $\quad \mu_{1}(\mathrm{x}) \leq \mu_{1}^{\prime}(\mathrm{h}(\mathrm{x})) ; \gamma_{1}(\mathrm{x}) \geq \gamma_{1}{ }^{\prime}(\mathrm{h}(\mathrm{x}))$ for every $\mathrm{x} \in \mathrm{S}$.

(ii) $\quad \mu_{2}(\mathrm{x}, \mathrm{y}) \leq \mu_{2}^{\prime}(\mathrm{h}(\mathrm{x}), \mathrm{h}(\mathrm{y})) ; \gamma_{2}(\mathrm{x}, \mathrm{y}) \geq \gamma_{2}(\mathrm{~h}(\mathrm{x}), \mathrm{h}(\mathrm{y}))$ for every $\mathrm{x} \in \mathrm{S}$

\section{Definition 2.10:}

A weak isomorphism of Intuitionistic fuzzy graphs $h: G \rightarrow G^{\prime}$ is a map $h: S \rightarrow S$ ' which is a bijective homomorphism that satisfies $\left(\mu_{1}(\mathrm{x}), \gamma_{1}(\mathrm{x})\right)=\left(\mu_{1}^{\prime}(\mathrm{h}(\mathrm{x})), \gamma_{1}{ }^{\prime}(\mathrm{h}(\mathrm{x}))\right.$ for every $\mathrm{x} C \mathrm{~S}$.

\section{Definition 2.11:}

A co-weak isomorphism of intuitionistic fuzzy graphs $h: G \rightarrow G^{\prime}$ is a map $h: S \rightarrow S$ ' which is a bijective homomorphism that satisfies $\left.\mu_{2}(\mathrm{x}, \mathrm{y}), \gamma_{2}(\mathrm{x}, \mathrm{y})\right)=\left(\mu_{2}{ }^{\prime}(\mathrm{h}(\mathrm{x}), \mathrm{h}(\mathrm{y})), \gamma_{2}{ }^{\prime}(\mathrm{h}(\mathrm{x}), \mathrm{h}(\mathrm{y}))\right)$ for every $\mathrm{x}, \mathrm{yCS}$.

Definition 2.12:

An isomorphism of intuitionistic fuzzy graphs $h: G \rightarrow G^{\prime}$ is a map $h: S \rightarrow S^{\prime}$ which is a bijective homomorphism that satisfies

(i) $\quad\left(\mu_{1}(\mathrm{x}), \gamma_{1}(\mathrm{x})\right)=\left(\mu_{1}^{\prime}(\mathrm{h}(\mathrm{x})), \gamma_{1}{ }^{\prime}(\mathrm{h}(\mathrm{x}))\right.$ for every $\mathrm{x} \in \mathrm{S}$.

(ii) $\quad\left(\mu_{2}(\mathrm{x}, \mathrm{y}), \gamma_{2}(\mathrm{x}, \mathrm{y})\right)=\left(\mu_{2}(\mathrm{~h}(\mathrm{x}), \mathrm{h}(\mathrm{y})), \gamma_{2}{ }^{\prime}(\mathrm{h}(\mathrm{x}), \mathrm{h}(\mathrm{y}))\right)$ for every $\mathrm{x}, \mathrm{y} \in \mathrm{S}$.

This will denote as $\mathrm{G} \cong \mathrm{G}^{\prime}$.

\section{Definition 2.13:}

An IntuitonisticFuzzy graph $\mathrm{G}$ is said to be self complementary if $\mathrm{G} \cong \bar{G}$.

Definition 2.14:

An Intuitonistic Fuzzy graphG is said to be self weak complementary if G is weak isomorphic with $\bar{G}$.

\section{Isomorphic properties of highly irregular IFG and its complement}

Definition 3.1Let $G=(V, E)$ be a connected IFG. Then, $G$ is said to be a highly irregular IFG if every vertex of $\mathrm{G}$ is adjacent to vertices with distinct degrees.

Theorem 3.2: Let $G$ and $G^{\prime}$ be two highly irregular Intuitionistic fuzzy graphs. If $G$ is co-weak isomorphic with $\mathrm{G}^{\prime}$, then there exists a homomorphism between $\bar{G}$ and $\bar{G}^{\prime}$, but the complements need not be highly irregular IFG.

Proof: Suppose G is co-weak isomorphis with $\mathrm{G}^{\prime}$, then $\mathrm{h}: \mathrm{S} \rightarrow \mathrm{S}^{\prime}$ is a bijective map that satisfies

$\mu_{1}(\mathrm{x}) \leq \mu_{1}^{\prime}(\mathrm{h}(\mathrm{x})) ; \gamma_{1}(\mathrm{x}) \geq \gamma_{1}{ }^{\prime}(\mathrm{h}(\mathrm{x}))$ for every $\mathrm{x} \in \mathrm{S}$. and

$\left(\mu 2(x, y), \gamma_{2}(x, y)\right)=\left(\mu 2^{\prime}(h(x), h(y)), \gamma_{2}^{\prime}(h(x), h(y))\right)$ for every $x, y \in S$.

In $\bar{G}, \bar{\mu}_{2}(\mathrm{x}, \mathrm{y})=\mu_{1}(\mathrm{x}) \Lambda \mu_{1}(\mathrm{y})-\mu_{2}(\mathrm{x}, \mathrm{y}) \leq \mu_{1}{ }^{\prime}(\mathrm{h}(\mathrm{x})) \Lambda \mu_{1}{ }^{\prime}(\mathrm{h}(\mathrm{y}))-\left(\mu 2^{\prime}(\mathrm{h}(\mathrm{x}), \mathrm{h}(\mathrm{y}))\right.$

$\leq \bar{\mu}_{2}{ }^{\prime}((\mathrm{h}(\mathrm{x}), \mathrm{h}(\mathrm{y}))$ for every $\mathrm{x}, \mathrm{y} \in \mathrm{S}$.

Similarly, $\bar{\gamma}_{2}(\mathrm{x}, \mathrm{y})=\gamma_{1}(\mathrm{x}) \mathrm{V} \gamma_{1}(\mathrm{y})-\gamma_{2}(\mathrm{x}, \mathrm{y}) \geq \gamma_{1}{ }^{\prime}(\mathrm{h}(\mathrm{x})) \mathrm{V} \gamma_{1}{ }^{\prime}(\mathrm{h}(\mathrm{y}))-\left(\gamma_{2}{ }^{\prime}(\mathrm{h}(\mathrm{x}), \mathrm{h}(\mathrm{y}))\right.$

$$
\geq \bar{\gamma}_{2}{ }^{\prime}((\mathrm{h}(\mathrm{x}), \mathrm{h}(\mathrm{y})) \text { for every } \mathrm{x}, \mathrm{y} \in \mathrm{CS} \text {. }
$$

Hence, we have $\mathrm{h}$ is a bijective homomorphism between $\bar{G}$ and $\bar{G}^{\prime}$. Also the complements need not be highly Irregular IFG

Proposition 3.3: If there is a co-weak isomorphism between two highly irregular Intuitionistic fuzzy graphs $G$ and $G^{\prime}$, then $\bar{G}$ and $\bar{G}^{\prime}$ need not be co-weak isomorphic. Also the complements need not highly Irregular IFG.

Example 3.4: $G=(V, E)$ be IFG with $V=\{a, b, c, d\}$ and defined by $\left(\mu_{1}(a), \gamma_{1}(a)\right)=(0.6,0.3)$,

$\left(\mu_{1}(\mathrm{~b}), \gamma_{1}(\mathrm{~b})\right)=(0.4,0.5),\left(\mu_{1}(\mathrm{c}), \gamma_{1}(\mathrm{c})\right)=(0.5,0.5),\left(\mu_{1}(\mathrm{~d}), \gamma_{1}(\mathrm{~d})\right)=(0.7,0.2),\left(\mu_{2}(\mathrm{a}, \mathrm{b}), \gamma_{2}(\mathrm{a}, \mathrm{b})\right)=(0.3,0.2)$,

$\left(\mu_{2}(b, c), \gamma_{2}(b, c)\right)=(0.2,0.4),\left(\mu_{2}(c, d), \gamma_{2}(c, d)\right)=(0.3,0.1),\left(\mu_{2}(d, a), \gamma_{2}(d, a)\right)=(0.5,0.2)$.

Then $\bar{G}$ will be $\left(\bar{\mu}_{1}(\mathrm{a}), \bar{\gamma}_{1}(\mathrm{a})\right)=(0.6,0.3),\left(\bar{\mu}_{1}(\mathrm{~b}), \bar{\gamma}_{1}(\mathrm{~b})\right)=(0.4,0.5),\left(\bar{\mu}_{1}(\mathrm{c}), \bar{\gamma}_{1}(\mathrm{c})\right)=(0.5,0.5)$,

$\left(\bar{\mu}_{1}(\mathrm{~d}), \bar{\gamma}_{1}(\mathrm{~d})\right)=(0.7,0.2)$ and $\left(\bar{\mu}_{2}(\mathrm{a}, \mathrm{b}), \bar{\gamma}_{2}(\mathrm{a}, \mathrm{b})\right)=(0.1,0.3),\left(\bar{\mu}_{2}(\mathrm{~b}, \mathrm{c}), \bar{\gamma}_{2}(\mathrm{~b}, \mathrm{c})\right)=(0.2,0.1)$,

$\left(\bar{\mu}_{2}(\mathrm{c}, \mathrm{d}), \bar{\gamma}_{2}(\mathrm{c}, \mathrm{d})\right)=(0.2,0.3),\left(\bar{\mu}_{2}(\mathrm{~d}, \mathrm{a}), \bar{\gamma}_{2}(\mathrm{~d}, \mathrm{a})\right)=(0.1,0.1),\left(\bar{\mu}_{2}(\mathrm{a}, \mathrm{c}), \bar{\gamma}_{2}(\mathrm{a}, \mathrm{c})\right)=(0.5,0.5),\left(\bar{\mu}_{2}(\mathrm{~b}, \mathrm{~d}), \bar{\gamma}_{2}(\mathrm{~b}, \mathrm{~d})\right)=$ $(0.4,0.5)$.

Also $G^{\prime}=\left(V^{\prime}, E^{\prime}\right)$ be IFG, with $V^{\prime}=\left\{a^{\prime}, b^{\prime}, c^{\prime}, d^{\prime}\right\}$ and defined by

$\left(\mu_{1}\left(a^{\prime}\right), \gamma_{1}\left(a^{\prime}\right)\right)=(0.6,0.3),\left(\mu_{1}\left(b^{\prime}\right), \gamma_{1}\left(b^{\prime}\right)\right)=(0.4,0.5),\left(\mu_{1}\left(c^{\prime}\right), \gamma_{1}\left(c^{\prime}\right)\right)=(0.8,0.2),\left(\mu_{1}\left(d^{\prime}\right), \gamma_{1}\left(d^{\prime}\right)\right)=(0.7,0.2)$, $\left(\mu_{2}\left(a^{\prime}, b^{\prime}\right), \gamma_{2}\left(a^{\prime}, b^{\prime}\right)\right)=(0.3,0.2),\left(\mu_{2}\left(b^{\prime}, c^{\prime}\right), \gamma_{2}\left(b^{\prime}, c^{\prime}\right)\right)=(0.2,0.4),\left(\mu_{2}\left(c^{\prime}, d^{\prime}\right), \gamma_{2}\left(c^{\prime}, d^{\prime}\right)\right)=(0.3,0.1)$,

$\left(\mu_{2}\left(d^{\prime}, a^{\prime}\right), \gamma_{2}\left(d^{\prime}, a^{\prime}\right)\right)=(0.5,0.2)$

Then $\bar{G}^{\prime}$ will be $\left(\bar{\mu}_{1}\left(\mathrm{a}^{\prime}\right), \bar{\gamma}_{1}\left(\mathrm{a}^{\prime}\right)\right)=(0.6,0.3),\left(\bar{\mu}_{1}\left(\mathrm{~b}^{\prime}\right), \bar{\gamma}_{1}\left(\mathrm{~b}^{\prime}\right)\right)=(0.4,0.5),\left(\bar{\mu}_{1}\left(\mathrm{c}^{\prime}\right), \bar{\gamma}_{1}\left(\mathrm{c}^{\prime}\right)\right)=(0.8,0.2)$,

$\left(\bar{\mu}_{1}\left(\mathrm{~d}^{\prime}\right), \bar{\gamma}_{1}\left(\mathrm{~d}^{\prime}\right)\right)=(0.7,0.2)$, and $\left(\bar{\mu}_{2}\left(\mathrm{a}^{\prime}, \mathrm{b}^{\prime}\right), \bar{\gamma}_{2}\left(\mathrm{a}^{\prime}, \mathrm{b}^{\prime}\right)\right)=(0.1,0.3),\left(\bar{\mu}_{2}\left(\mathrm{~b}^{\prime}, \mathrm{c}^{\prime}\right), \bar{\gamma}_{2}\left(\mathrm{~b}^{\prime}, \mathrm{c}^{\prime}\right)\right)=(0.2,0.1)$,

$\left(\bar{\mu}_{2}\left(\mathrm{c}^{\prime}, \mathrm{d}^{\prime}\right), \bar{\gamma}_{2}\left(\mathrm{c}^{\prime}, \mathrm{d}^{\prime}\right)\right)=(0.4,0.1),\left(\bar{\mu}_{2}\left(\mathrm{~d}^{\prime}, \mathrm{a}^{\prime}\right), \bar{\gamma}_{2}\left(\mathrm{~d}^{\prime}, \mathrm{a}^{\prime}\right)\right)=(0.1,0.1),\left(\bar{\mu}_{2}\left(\mathrm{a}^{\prime}, \mathrm{c}^{\prime}\right), \bar{\gamma}_{2}\left(\mathrm{a}^{\prime}, \mathrm{c}^{\prime}\right)\right)=(0.6,0.3)$,

$\left(\bar{\mu}_{2}\left(\mathrm{~b}^{\prime}, \mathrm{d}^{\prime}\right), \bar{\gamma}_{2}\left(\mathrm{~b}^{\prime}, \mathrm{d}^{\prime}\right)\right)=(0.4,0.5)$. 
In this example, $\mathrm{G}$ is co-weak isomorphic with $\mathrm{G}^{\prime}$, But $\bar{G}$ is not co-weak isomorphic with $\bar{G}^{\prime}$. However there is homomorphism between $\bar{G}$ and $\bar{G}^{\prime}$. Also $\bar{G}$ is not highly irregular IFG because for vertices c and $\mathrm{d}$, the adjacent vertices a and b are having same degrees. But $\bar{G}^{\prime}$ is highly irregular IFG.

\section{Theorem 3.5:}

A highly Irregular IFG need not be self complementary.

Proof: In the complement IFG, to every vertex, the adjacent vertices with distinct degree or the non- adjacent vertices with distinct or same degrees may happen to be adjacent vertices with same degrees. So the complement may or may not highly irregular IFG.

\section{Example 3.5:}

$\mathrm{G}=(\mathrm{V}, \mathrm{E})$ be IFG with $\mathrm{V}=\{\mathrm{a}, \mathrm{b}, \mathrm{c}, \mathrm{d}\}$ and defined by $\left(\mu_{1}(\mathrm{a}), \gamma_{1}(\mathrm{a})\right)=(0.5,0.3),\left(\mu_{1}(\mathrm{~b}), \gamma_{1}(\mathrm{~b})\right)=(0.4,0.2)$, $\left(\mu_{1}(\mathrm{c}), \gamma_{1}(\mathrm{c})\right)=(0.7,0.3),\left(\mu_{1}(\mathrm{~d}), \gamma_{1}(\mathrm{~d})\right)=(0.8,0.1),\left(\mu_{2}(\mathrm{a}, \mathrm{b}), \gamma_{2}(\mathrm{a}, \mathrm{b})\right)=(0.4,0.3),\left(\mu_{2}(\mathrm{~b}, \mathrm{c}), \gamma_{2}(\mathrm{~b}, \mathrm{c})\right)=(0.4,0.3)$, $\left(\mu_{2}(\mathrm{c}, \mathrm{d}), \gamma_{2}(\mathrm{c}, \mathrm{d})\right)=(0.4,0.1),\left(\mu_{2}(\mathrm{~d}, \mathrm{a}), \gamma_{2}(\mathrm{~d}, \mathrm{a})\right)=(0.5,0.3)\left(\mu_{2}(\mathrm{~b}, \mathrm{~d}), \gamma_{2}(\mathrm{~b}, \mathrm{~d})\right)=(0.2,0.1)$.

Then $\bar{G}$ will be $\left(\bar{\mu}_{1}(\mathrm{a}), \bar{\gamma}_{1}(\mathrm{a})\right)=(0.5,0.3),\left(\bar{\mu}_{1}(\mathrm{~b}), \bar{\gamma}_{1}(\mathrm{~b})\right)=(0.4,0.2),\left(\bar{\mu}_{1}(\mathrm{c}), \bar{\gamma}_{1}(\mathrm{c})\right)=(0.7,0.3),\left(\bar{\mu}_{1}(\mathrm{~d}), \bar{\gamma}_{1}(\mathrm{~d})\right)=$ $(0.8,0.1)$ and $\left(\bar{\mu}_{2}(\mathrm{c}, \mathrm{d}), \bar{\gamma}_{2}(\mathrm{c}, \mathrm{d})\right)=(0.3,0.2),\left(\bar{\mu}_{2}(\mathrm{a}, \mathrm{c}), \bar{\gamma}_{2}(\mathrm{a}, \mathrm{c})\right)=(0.5,0.3),\left(\bar{\mu}_{2}(\mathrm{~b}, \mathrm{~d}), \bar{\gamma}_{2}(\mathrm{~b}, \mathrm{~d})\right)=(0.2,0.1)$.

Here, $\mathrm{G}$ is highly Irregular IFG but not self complementary highly Irregular IFG.

\section{Example3.6:}

$\mathrm{G}=(\mathrm{V}, \mathrm{E})$ be IFG with $\mathrm{V}=\{\mathrm{a}, \mathrm{b}, \mathrm{c}, \mathrm{d}\}$ and defined by $\left(\mu_{1}(\mathrm{a}), \gamma_{1}(\mathrm{a})\right)=(0.4,0.5),\left(\mu_{1}(\mathrm{~b}), \gamma_{1}(\mathrm{~b})\right)=(0.4,0.6)$, $\left(\mu_{1}(\mathrm{c}), \gamma_{1}(\mathrm{c})\right)=(0.8,0.2),\left(\mu_{1}(\mathrm{~d}), \gamma_{1}(\mathrm{~d})\right)=(0.6,0.3),\left(\mu_{2}(\mathrm{a}, \mathrm{b}), \gamma_{2}(\mathrm{a}, \mathrm{b})\right)=(0.1,0.4),\left(\mu_{2}(\mathrm{~b}, \mathrm{c}), \gamma_{2}(\mathrm{~b}, \mathrm{c})\right)=(0.2,0.1)$, $\left(\mu_{2}(\mathrm{c}, \mathrm{d}), \gamma_{2}(\mathrm{c}, \mathrm{d})\right)=(0.2,0.1),\left(\mu_{2}(\mathrm{~d}, \mathrm{a}), \gamma_{2}(\mathrm{~d}, \mathrm{a})\right)=(0.3,0.2)$.

Then $\bar{G}$ will be $\left(\bar{\mu}_{1}(\mathrm{a}), \bar{\gamma}_{1}(\mathrm{a})\right)=(0.4,0.5),\left(\bar{\mu}_{1}(\mathrm{~b}), \bar{\gamma}_{1}(\mathrm{~b})\right)=(0.4,0.6),\left(\bar{\mu}_{1}(\mathrm{c}), \bar{\gamma}_{1}(\mathrm{c})\right)=(0.8,0.2),\left(\bar{\mu}_{1}(\mathrm{~d}), \bar{\gamma}_{1}(\mathrm{~d})\right)=$ $(0.6,0.3)$ and $\left(\bar{\mu}_{2}(\mathrm{a}, \mathrm{b}), \bar{\gamma}_{2}(\mathrm{a}, \mathrm{b})\right)=(0.3,0.2),\left(\bar{\mu}_{2}(\mathrm{~b}, \mathrm{c}), \bar{\gamma}_{2}(\mathrm{~b}, \mathrm{c})\right)=(0.2,0.5),\left(\bar{\mu}_{2}(\mathrm{c}, \mathrm{d}), \bar{\gamma}_{2}(\mathrm{c}, \mathrm{d})\right)=(0.4,0.2)$,

$\left(\bar{\mu}_{2}(\mathrm{~d}, \mathrm{a}), \bar{\gamma}_{2}(\mathrm{~d}, \mathrm{a})\right)=(0.1,0.3),\left(\bar{\mu}_{2}(\mathrm{a}, \mathrm{c}), \bar{\gamma}_{2}(\mathrm{a}, \mathrm{c})\right)=(0.4,0.5),\left(\bar{\mu}_{2}(\mathrm{~b}, \mathrm{~d}), \bar{\gamma}_{2}(\mathrm{~b}, \mathrm{~d})\right)=(0.4,0.6)$.

Here $G$ and $\bar{G}$ are highly Irregular IFG, but $G$ is not weak isomorphic with $\bar{G}$. Hence $G$ is not a self weak complementary highly Irregular IFG.

\section{Example 3.7:}

$\mathrm{G}=(\mathrm{V}, \mathrm{E})$ be IFG with $\mathrm{V}=\{\mathrm{a}, \mathrm{b}, \mathrm{c}, \mathrm{d}\}$ and defined by $\left(\mu_{1}(\mathrm{a}), \gamma_{1}(\mathrm{a})\right)=(0.5,0.4),\left(\mu_{1}(\mathrm{~b}), \gamma_{1}(\mathrm{~b})\right)=(0.4,0.2)$,

$\left(\mu_{1}(\mathrm{c}), \gamma_{1}(\mathrm{c})\right)=(0.7,0.3),\left(\mu_{1}(\mathrm{~d}), \gamma_{1}(\mathrm{~d})\right)=(0.8,0.2),\left(\mu_{2}(\mathrm{a}, \mathrm{b}), \gamma_{2}(\mathrm{a}, \mathrm{b})\right)=(0.2,0.2),\left(\mu_{2}(\mathrm{~b}, \mathrm{c}), \gamma_{2}(\mathrm{~b}, \mathrm{c})\right)=(0.2,0.2)$, $\left(\mu_{2}(\mathrm{c}, \mathrm{d}), \gamma_{2}(\mathrm{c}, \mathrm{d})\right)=(0.3,0.2),\left(\mu_{2}(\mathrm{~d}, \mathrm{a}), \gamma_{2}(\mathrm{~d}, \mathrm{a})\right)=(0.2,0.3),\left(\mu_{2}(\mathrm{a}, \mathrm{c}), \gamma_{2}(\mathrm{a}, \mathrm{c})\right)=(0.5,0.4)$

Then $\bar{G}$ will be $\left(\bar{\mu}_{1}(\mathrm{a}), \bar{\gamma}_{1}(\mathrm{a})\right)=(0.5,0.4),\left(\bar{\mu}_{1}(\mathrm{~b}), \bar{\gamma}_{1}(\mathrm{~b})\right)=(0.4,0.2),\left(\bar{\mu}_{1}(\mathrm{c}), \bar{\gamma}_{1}(\mathrm{c})\right)=(0.7,0.3)$,

$\left(\bar{\mu}_{1}(\mathrm{~d}), \bar{\gamma}_{1}(\mathrm{~d})\right)=(0.8,0.2),\left(\bar{\mu}_{2}(\mathrm{a}, \mathrm{b}), \bar{\gamma}_{2}(\mathrm{a}, \mathrm{b})\right)=(0.2,0.1),\left(\bar{\mu}_{2}(\mathrm{~b}, \mathrm{c}), \bar{\gamma}_{2}(\mathrm{~b}, \mathrm{c})\right)=(0.2,0.1),\left(\bar{\mu}_{2}(\mathrm{c}, \mathrm{d}), \bar{\gamma}_{2}(\mathrm{c}, \mathrm{d})\right)=(0.4,0.1)$, $\left(\bar{\mu}_{2}(\mathrm{~d}, \mathrm{a}), \bar{\gamma}_{2}(\mathrm{~d}, \mathrm{a})\right)=(0.3,0.1),\left(\bar{\mu}_{2}(\mathrm{~b}, \mathrm{~d}), \bar{\gamma}_{2}(\mathrm{~b}, \mathrm{~d})\right)=(0.4,0.2)$.

Here $G$ and $\bar{G}$ are highly Irregular IFG, and $G$ is weak isomorphic with $\bar{G}$. Hence $G$ is a self weak complementary highly Irregular IFG.

Theorem 3.8: Let $\mathrm{G}$ be self weak complementary highly Irregular IFG, then

$\sum_{x \neq y} \mu_{2}(x, y) \leq \frac{1}{2} \sum_{x \neq y} \mu_{1}(x) \Lambda \mu_{1}(y)$ and $\sum_{x \neq y} \gamma_{2}(x, y) \geq \frac{1}{2} \sum_{x \neq y} \gamma_{1}(x) \vee \gamma_{1}(y)$

Proof:

Given $\mathrm{G}$ is self weak complementary IFG then $\mathrm{G}$ is weak isomorphic with $\bar{G}$.

Therefore the weak isomorphism of intuitionistic fuzzy graphs $h: G \rightarrow \bar{G}$ is a map $h: S \rightarrow S$ which is a bijective homomorphism that satisfies $\left(\mu_{1}(\mathrm{x}), \gamma_{1}(\mathrm{x})\right)=\left(\overline{\mu_{1}}(\mathrm{~h}(\mathrm{x})), \overline{\gamma_{1}}(\mathrm{~h}(\mathrm{x}))\right.$ for every $\mathrm{x} \in \mathrm{S}$

And $\mu_{2}(\mathrm{x}, \mathrm{y}) \leq \overline{\mu_{2}}(\mathrm{~h}(\mathrm{x}), \mathrm{h}(\mathrm{y})) ; \gamma_{2}(\mathrm{x}, \mathrm{y}) \geq \overline{\gamma_{2}}(\mathrm{~h}(\mathrm{x}), \mathrm{h}(\mathrm{y}))$ for every $\mathrm{x} \in \mathrm{S}$.

Now by using definition of complement,

$\mu_{2}(\mathrm{x}, \mathrm{y}) \leq \mu_{1}(\mathrm{~h}(\mathrm{x})) \Lambda \mu_{1}(\mathrm{~h}(\mathrm{y}))-\mu_{2}(\mathrm{~h}(\mathrm{x}), \mathrm{h}(\mathrm{y}))=\mu_{1}(\mathrm{x}) \Lambda \mu_{1}(\mathrm{y})-\mu_{2}(\mathrm{~h}(\mathrm{x}), \mathrm{h}(\mathrm{y}))$

i.e) $\mu_{2}(\mathrm{x}, \mathrm{y})+\mu_{2}(\mathrm{~h}(\mathrm{x}), \mathrm{h}(\mathrm{y})) \leq \mu_{1}(\mathrm{x}) \Lambda \mu_{1}(\mathrm{y})$

Taking summation on both sides

$\sum_{x \neq y} \mu_{2}(x, y)+\sum_{x \neq y} \mu_{2}(\mathrm{~h}(\mathrm{x}), \mathrm{h}(\mathrm{y})) \leq \sum_{x \neq y} \mu_{1}(\mathrm{x}) \Lambda \mu_{1}(\mathrm{y})$

Since $\mathrm{S}$ is finite set, we have

$2 \sum_{x \neq y} \mu_{2}(x, y) \leq \sum_{x \neq y} \mu_{1}(\mathrm{x}) \Lambda \mu_{1}(\mathrm{y})$

$\sum_{x \neq y} \mu_{2}(x, y) \leq 1 / 2 \sum_{x \neq y} \mu_{1}(\mathrm{x}) \Lambda \mu_{1}(\mathrm{y})$ for every $\mathrm{x}, \mathrm{y}$ CS.

Similarly,

$$
\begin{aligned}
& \gamma_{2}(\mathrm{x}, \mathrm{y}) \geq \gamma_{1}(\mathrm{~h}(\mathrm{x})) \Lambda \gamma_{1}(\mathrm{~h}(\mathrm{y}))-\gamma_{2}(\mathrm{~h}(\mathrm{x}), \mathrm{h}(\mathrm{y}))=\gamma_{1}(\mathrm{x}) \mathrm{V} \gamma_{1}(\mathrm{y})-\gamma_{2}(\mathrm{~h}(\mathrm{x}), \mathrm{h}(\mathrm{y})) \\
& \Rightarrow \gamma_{2}(\mathrm{x}, \mathrm{y})+\gamma_{2}(\mathrm{~h}(\mathrm{x}), \mathrm{h}(\mathrm{y})) \geq \gamma_{1}(\mathrm{x}) \mathrm{V} \gamma_{1}(\mathrm{y}) \\
& \quad \Rightarrow \sum_{x \neq y} \gamma_{2}(x, y)+\sum_{x \neq y} \gamma_{2}(\mathrm{~h}(\mathrm{x}), \mathrm{h}(\mathrm{y})) \geq \sum_{x \neq y} \gamma_{1}(\mathrm{x}) \mathrm{V} \gamma_{1}(\mathrm{y})
\end{aligned}
$$


$\Rightarrow 2 \sum_{x \neq y} \gamma_{2}(x, y) \geq \sum_{x \neq y} \gamma_{1}(\mathrm{x}) \mathrm{V} \gamma_{1}(\mathrm{y})$

Hence, $\sum_{x \neq y} \gamma_{2}(x, y) \geq 1 / 2 \sum_{x \neq y} \gamma_{1}(\mathrm{x}) \mathrm{V} \gamma_{1}$ (y)for every x,y $\in \mathrm{S}$.

IV. Isomorphic properties of Neighbourly Irregular IFG and its complement

Definition 4.1: Let $G=\langle\mathrm{V}, \mathrm{E}>$ be a connected IFG. $\mathrm{G}$ is said to be a neighbourly irregular fuzzy graph if every two adjacent vertices of $\mathrm{G}$ have distinct degree.

Theorem 4.2: Let $\mathrm{G}=(\mathrm{V}, \mathrm{E})$ and $\mathrm{G}^{\prime}=\left(\mathrm{V}^{\prime}, \mathrm{E}^{\prime}\right)$ be two neighbourly irregular intuitionistic fuzzy graphs, $\mathrm{G}$ and $\mathrm{G}$ ' are isomorphic if and only if their complements are isomorphic, but the complements need not be neighbourly irregular IFG.

Proof: $L$ et $G=(V, E)$ and $G^{\prime}=\left(V^{\prime}, E^{\prime}\right)$ be two neighbourly irregular intuitionistic fuzzy graphs.

Assume $\mathrm{G} \cong \mathrm{G}$.

(i.e) There exists a bijective map h: $S \rightarrow S^{\prime}$ satisfying $\left(\mu_{1}(x), \gamma_{1}(x)\right)=\left(\mu_{1}^{\prime}(h(x)), \gamma_{1}{ }^{\prime}(h(x))\right.$ for every $x \in S$ and $\left(\mu_{2}(\mathrm{x}, \mathrm{y}), \gamma_{2}(\mathrm{x}, \mathrm{y})\right)=\left(\mu_{2}{ }^{\prime}(\mathrm{h}(\mathrm{x}), \mathrm{h}(\mathrm{y})), \gamma_{2}{ }^{\prime}(\mathrm{h}(\mathrm{x}), \mathrm{h}(\mathrm{y}))\right)$ for every $\mathrm{x}, \mathrm{y} \in \mathrm{S}$.

By the definition of complement of IFG, $\left(\bar{\mu}_{1}(x), \bar{\gamma}_{1}(x)\right)=\left(\bar{\mu}_{1}{ }^{\prime}\left(\mathrm{h}(\mathrm{x}), \bar{\gamma}_{1}{ }^{\prime}(\mathrm{h}(\mathrm{x}))\right.\right.$ and

$\left(\bar{\mu}_{2}(\mathrm{x}, \mathrm{y}), \bar{\gamma}_{2}(\mathrm{x}, \mathrm{y})\right)=\left(\mu_{1}(\mathrm{x}) \Lambda \mu_{1}(\mathrm{y})-\mu_{2}(\mathrm{x}, \mathrm{y}), \gamma_{1}(\mathrm{x}) \mathrm{V} \gamma_{1}(\mathrm{y})-\gamma_{2}(\mathrm{x}, \mathrm{y})\right)$

$=\left(\mu_{1}{ }^{\prime}(\mathrm{h}(\mathrm{x})) \Lambda \mu_{1}^{\prime}(\mathrm{h}(\mathrm{y}))-\mu_{2}{ }^{\prime}\left((\mathrm{h}(\mathrm{x}), \mathrm{h}(\mathrm{y})), \gamma_{1}{ }^{\prime}(\mathrm{h}(\mathrm{x})) \mathrm{V} \gamma_{1}{ }^{\prime}(\mathrm{h}(\mathrm{y}))-\gamma_{2}{ }^{\prime}((\mathrm{h}(\mathrm{x}), \mathrm{h}(\mathrm{y}))\right.\right.$

$=\left(\bar{\mu}_{2}{ }^{\prime}(\mathrm{h}(\mathrm{x}), \mathrm{h}(\mathrm{y})), \bar{\gamma}_{2}{ }^{\prime}((\mathrm{h}(\mathrm{x}), \mathrm{h}(\mathrm{y})))\right.$ for every $\mathrm{x}, \mathrm{y} \in \mathrm{S}$. which implies $\bar{G} \cong \bar{G}$ '.

But the complements need not be neighbourly irregular.

Conversely, assume that $\bar{G} \cong \bar{G}^{\prime}$. i.e)there exist a bijective map g: $\mathrm{S} \rightarrow \mathrm{S}$ ' satisfying

$\left(\mu_{1}(\mathrm{x}), \gamma_{1}(\mathrm{x})\right)=\left(\mu_{1}^{\prime}(\mathrm{g}(\mathrm{x})), \gamma_{1}^{\prime}(\mathrm{g}(\mathrm{x}))\right.$ for every $\mathrm{x} \in \mathrm{S}$ and

$\left(\bar{\mu}_{2}(\mathrm{x}, \mathrm{y}), \bar{\gamma}_{2}(\mathrm{x}, \mathrm{y})\right)=\left(\bar{\mu}_{2}{ }^{\prime}(\mathrm{g}(\mathrm{x}), \mathrm{g}(\mathrm{y})), \bar{\gamma}_{2}{ }^{\prime}((\mathrm{g}(\mathrm{x}), \mathrm{g}(\mathrm{y})))\right.$ for every $\mathrm{x}, \mathrm{y} \in \mathrm{S}$

Using definition of complement,

$\left(\bar{\mu}_{2}(\mathrm{x}, \mathrm{y}), \bar{\gamma}_{2}(\mathrm{x}, \mathrm{y})\right)=\left(\mu_{1}(\mathrm{x}) \Lambda \mu_{1}(\mathrm{y})-\mu_{2}(\mathrm{x}, \mathrm{y}), \gamma_{1}(\mathrm{x}) \vee \gamma_{1}(\mathrm{y})-\gamma_{2}(\mathrm{x}, \mathrm{y})\right)$ for every $\mathrm{x}, \mathrm{y} \in \mathrm{S}$

$\left(\bar{\mu}_{2}^{\prime}(\mathrm{g}(\mathrm{x}), \mathrm{g}(\mathrm{y})), \bar{\gamma}_{2}{ }^{\prime}((\mathrm{g}(\mathrm{x}), \mathrm{g}(\mathrm{y})))=\left(\mu_{1}{ }^{\prime}(\mathrm{g}(\mathrm{x})) \Lambda \mu_{1}{ }^{\prime}(\mathrm{g}(\mathrm{y}))-\mu_{2}{ }^{\prime}\left((\mathrm{g}(\mathrm{x}), \mathrm{g}(\mathrm{y})), \gamma_{1}{ }^{\prime}(\mathrm{g}(\mathrm{x})) \mathrm{V}\right.\right.\right.$

$\gamma_{1}^{\prime}(\mathrm{g}(\mathrm{y}))-\gamma_{2}{ }^{\prime}((\mathrm{g}(\mathrm{x}), \mathrm{g}(\mathrm{y}))$ for every $\mathrm{x}, \mathrm{y} \in \mathrm{S}$

Using the previous two equations, we get

$\left(\mu_{2}(x, y), \gamma_{2}(x, y)\right)=\left(\mu_{2}^{\prime}(g(x), g(y)), \gamma_{2}^{\prime}(g(x), g(y))\right)$ for every $x, y \in S$

Hence, $g: S \rightarrow S^{\prime}$ is an isomorphism between $G$ and $G^{\prime}$. (i.e) $G \cong G$ '.

Example 4.3:Consider two neighbourly irregular Intutitionistic fuzzy graphs $G=(V, E)$ and $G^{\prime}=\left(V^{\prime}, E^{\prime}\right)$ defined by $\left(\mu_{1}(\mathrm{a}), \gamma_{1}(\mathrm{a})\right)=(0.6,0.3),\left(\mu_{1}(\mathrm{~b}), \gamma_{1}(\mathrm{~b})\right)=(0.5,0.4),\left(\mu_{1}(\mathrm{c}), \gamma_{1}(\mathrm{c})\right)=(0.6,0.3),\left(\mu_{1}(\mathrm{~d}), \gamma_{1}(\mathrm{~d})\right)=(0.8,0.2)$, $\left(\mu_{2}(\mathrm{a}, \mathrm{b}), \gamma_{2}(\mathrm{a}, \mathrm{b})\right)=(0.3,0.2),\left(\mu_{2}(\mathrm{~b}, \mathrm{c}), \gamma_{2}(\mathrm{~b}, \mathrm{c})\right)=(0.3,0.2),\left(\mu_{2}(\mathrm{c}, \mathrm{d}), \gamma_{2}(\mathrm{c}, \mathrm{d})\right)=(0.4,0.1),\left(\mu_{2}(\mathrm{~d}, \mathrm{a}), \gamma_{2}(\mathrm{~d}, \mathrm{a})\right)=$ $(0.4,0.1)$ and $\left(\mu_{1}\left(\mathrm{a}^{\prime}\right), \gamma_{1}\left(\mathrm{a}^{\prime}\right)\right)=(0.6,0.3),\left(\mu_{1}\left(\mathrm{~b}^{\prime}\right), \gamma_{1}\left(\mathrm{~b}^{\prime}\right)\right)=(0.5,0.4),\left(\mu_{1}\left(\mathrm{c}^{\prime}\right), \gamma_{1}\left(\mathrm{c}^{\prime}\right)\right)=(0.6,0.3)$,

$\left(\mu_{1}\left(d^{\prime}\right), \gamma_{1}\left(d^{\prime}\right)\right)=(0.8,0.2),\left(\mu_{2}\left(a^{\prime}, b^{\prime}\right), \gamma_{2}\left(a^{\prime}, b^{\prime}\right)\right)=(0.3,0.2),\left(\mu_{2}\left(b^{\prime}, c^{\prime}\right), \gamma_{2}\left(b^{\prime}, c^{\prime}\right)\right)=(0.3,0.2)$,

$\left(\mu_{2}\left(c^{\prime}, d^{\prime}\right), \gamma_{2}\left(c^{\prime}, d^{\prime}\right)\right)=(0.4,0.1),\left(\mu_{2}\left(d^{\prime}, a^{\prime}\right), \gamma_{2}\left(d^{\prime}, a^{\prime}\right)\right)=(0.4,0.1)$.

Then complement of $\mathrm{G}$ and $\mathrm{G}$ ' are $\bar{G}$ and $\bar{G}^{\prime}$ will be

$\left(\bar{\mu}_{1}(\mathrm{a}), \bar{\gamma}_{1}(\mathrm{a})\right)=(0.6,0.3),\left(\bar{\mu}_{1}(\mathrm{~b}), \bar{\gamma}_{1}(\mathrm{~b})\right)=(0.5,0.4),\left(\bar{\mu}_{1}(\mathrm{c}), \gamma_{1}(\mathrm{c})\right)=(0.6,0.3),\left(\bar{\mu}_{1}(\mathrm{~d}), \bar{\gamma}_{1}(\mathrm{~d})\right)=(0.8,0.2)$,

$\left(\bar{\mu}_{2}(\mathrm{a}, \mathrm{b}), \bar{\gamma}_{2}(\mathrm{a}, \mathrm{b})\right)=(0.2,0.2),\left(\bar{\mu}_{2}(\mathrm{~b}, \mathrm{c}), \bar{\gamma}_{2}(\mathrm{~b}, \mathrm{c})\right)=(0.2,0.2),\left(\bar{\mu}_{2}(\mathrm{c}, \mathrm{d}), \bar{\gamma}_{2}(\mathrm{c}, \mathrm{d})\right)=(0.2,0.2),\left(\bar{\mu}_{2}(\mathrm{~d}, \mathrm{a}), \bar{\gamma}_{2}(\mathrm{~d}, \mathrm{a})\right)=$ $(0.2,0.2),\left(\bar{\mu}_{2}(\mathrm{a}, \mathrm{c}), \bar{\gamma}_{2}(\mathrm{a}, \mathrm{c})\right)=(0.6,0.3),\left(\bar{\mu}_{2}(\mathrm{~b}, \mathrm{~d}), \bar{\gamma}_{2}(\mathrm{~b}, \mathrm{~d})\right)=(0.5,0.4)$ and $\left(\bar{\mu}_{1}\left(\mathrm{a}^{\prime}\right), \bar{\gamma}_{1}\left(\mathrm{a}^{\prime}\right)\right)=(0.6,0.3)$,

$\left(\bar{\mu}_{1}\left(\mathrm{~b}^{\prime}\right), \bar{\gamma}_{1}\left(\mathrm{~b}^{\prime}\right)\right)=(0.5,0.4),\left(\bar{\mu}_{1}\left(\mathrm{c}^{\prime}\right), \bar{\gamma}_{1}\left(\mathrm{c}^{\prime}\right)\right)=(0.6,0.3),\left(\bar{\mu}_{1}\left(\mathrm{~d}^{\prime}\right), \bar{\gamma}_{1}\left(\mathrm{~d}^{\prime}\right)\right)=(0.8,0.2),\left(\bar{\mu}_{2}\left(\mathrm{a}^{\prime}, \mathrm{b}^{\prime}\right), \bar{\gamma}_{2}\left(\mathrm{a}^{\prime}, \mathrm{b}^{\prime}\right)\right)=$ $(0.2,0.2),\left(\bar{\mu}_{2}\left(\mathrm{~b}^{\prime}, \mathrm{c}^{\prime}\right), \bar{\gamma}_{2}\left(\mathrm{~b}^{\prime}, \mathrm{c}^{\prime}\right)\right)=(0.2,0.2),\left(\bar{\mu}_{2}\left(\mathrm{c}^{\prime}, \mathrm{d}^{\prime}\right), \bar{\gamma}_{2}\left(\mathrm{c}^{\prime}, \mathrm{d}^{\prime}\right)\right)=(0.2,0.2),\left(\bar{\mu}_{2}\left(\mathrm{~d}^{\prime}, \mathrm{a}^{\prime}\right), \bar{\gamma}_{2}\left(\mathrm{~d}^{\prime}, \mathrm{a}^{\prime}\right)\right)=(0.2,0.2)$, $\left(\bar{\mu}_{2}\left(\mathrm{a}^{\prime}, \mathrm{c}^{\prime}\right), \bar{\gamma}_{2}\left(\mathrm{a}^{\prime}, \mathrm{c}^{\prime}\right)\right)=(0.6,0.3),\left(\bar{\mu}_{2}\left(\mathrm{~b}^{\prime}, \mathrm{d}^{\prime}\right), \bar{\gamma}_{2}\left(\mathrm{~b}^{\prime}, \mathrm{d}^{\prime}\right)\right)=(0.5,0.4)$.

In this example, $\mathrm{G}$ is isomorphic with $\mathrm{G}^{\prime}$, and $\bar{G}$ is also isomorphic with $\bar{G}^{\prime}$. But the complements are not neighbourly irregular IFG.

Theorem 4.4: Let $\mathrm{G}=(\mathrm{V}, \mathrm{E})$ and $\mathrm{G}^{\prime}=\left(\mathrm{V}^{\prime}, \mathrm{E}^{\prime}\right)$ be two neighbourly irregular intuitionistic fuzzy graphs, $\mathrm{G}$ is weak isomorphic with $\mathrm{G}^{\prime}$, then $\bar{G}$ ' is weak isomorphic with $\bar{G}$, but the complements need not be neighbourly irregular.

Proof: If $h$ is a weak isomorphism between $G$ and $\mathrm{G}^{\prime}$, then $\mathrm{h}: \mathrm{S} \rightarrow \mathrm{S}^{\prime}$ is a bijective map that satisfies

$\mathrm{h}(\mathrm{x})=\mathrm{x}^{\prime}, \mathrm{x} \in \mathrm{S},\left(\mu_{1}(\mathrm{x}), \gamma_{1}(\mathrm{x})\right)=\left(\mu_{1}^{\prime}(\mathrm{h}(\mathrm{x})), \gamma_{1}^{\prime}(\mathrm{h}(\mathrm{x}))\right.$ for every $\mathrm{x} \in \mathrm{S}$

Also $\mu_{2}(\mathrm{x}, \mathrm{y}) \leq \mu_{2}{ }^{\prime}(\mathrm{h}(\mathrm{x}), \mathrm{h}(\mathrm{y})) ; \gamma_{2}(\mathrm{x}, \mathrm{y}) \geq \gamma_{2}{ }^{\prime}(\mathrm{h}(\mathrm{x}), \mathrm{h}(\mathrm{y}))$ for every $\mathrm{x}, \mathrm{y} \in \mathrm{S}$

As $h^{-1}: S^{\prime} \rightarrow S$ is also bijective for every $x^{\prime} \in S^{\prime}$, there is and $x \in S$ such that $h^{-1}\left(x^{\prime}\right)=x$

Using this in $(1),\left(\mu_{1}{ }^{\prime}\left(x^{\prime}\right), \gamma_{1}^{\prime}\left(x^{\prime}\right)\right)=\left(\mu_{1}\left(h^{-1}\left(x^{\prime}\right)\right), \gamma_{1}\left(h^{-1}\left(x^{\prime}\right)\right)\right)$ for every $x^{\prime} \in S^{\prime}$

Also by using in (2), $\bar{\mu}_{2}\left(\mathrm{~h}^{-1}\left(\mathrm{x}^{\prime}\right), \mathrm{h}^{-1}\left(\mathrm{y}^{\prime}\right)\right) \geq \mu_{1}(\mathrm{~h}(\mathrm{x})) \Lambda \mu_{1}^{\prime}(\mathrm{h}(\mathrm{y}))-\mu_{2}{ }^{\prime}(\mathrm{h}(\mathrm{x}), \mathrm{h}(\mathrm{y}))$

$$
\begin{aligned}
& =\mu_{1}{ }^{\prime}\left(x^{\prime}\right) \Lambda \mu_{1}^{\prime}\left(y^{\prime}\right)-\mu_{2}{ }^{\prime}\left(x^{\prime}, y^{\prime}\right) \\
& =\bar{\mu}_{2}{ }^{\prime}\left(x^{\prime}, y^{\prime}\right)
\end{aligned}
$$

(i.e) $\bar{\mu}_{2}^{\prime}\left(x^{\prime}, y^{\prime}\right) \leq \bar{\mu}_{2}\left(h^{-1}\left(x^{\prime}\right), h^{-1}\left(y^{\prime}\right)\right)$ 


$$
\text { Similarly, } \begin{aligned}
\bar{\gamma}_{2}\left(\mathrm{~h}^{-1}\left(\mathrm{x}^{\prime}\right), \mathrm{h}^{-1}\left(\mathrm{y}^{\prime}\right)\right) \leq & \gamma_{1}{ }^{\prime}(\mathrm{h}(\mathrm{x})) \mathrm{V} \gamma_{1}{ }^{\prime}(\mathrm{h}(\mathrm{y}))-\gamma_{2}{ }^{\prime}((\mathrm{h}(\mathrm{x}), \mathrm{h}(\mathrm{y})) \\
& =\gamma_{1}{ }^{\prime}\left(\mathrm{x}^{\prime}\right) \mathrm{V} \gamma_{1}{ }^{\prime}\left(\mathrm{y}^{\prime}\right)-\gamma_{2}{ }^{\prime}\left(\mathrm{x}^{\prime}, \mathrm{y}^{\prime}\right) \\
& =\bar{\gamma}_{2}^{\prime}\left(\mathrm{x}^{\prime}, \mathrm{y}^{\prime}\right) \text { for every } \mathrm{x}^{\prime}, \mathrm{y}^{\prime} \in \mathrm{S}^{\prime}
\end{aligned}
$$

Thus, $\mathrm{h}^{-1}: \mathrm{S}^{\prime} \rightarrow \mathrm{S}$ is a bijective map which is a weak isomorphism between $\bar{G}$, and $\bar{G}$. But the complements need not be neighbourlyIrregular Intuitionistic fuzzy graphs.

\section{Example 4.5:}

Consider two neighbourly irregular Intutitionistic fuzzy graphs $\mathrm{G}=(\mathrm{V}, \mathrm{E})$ and $\mathrm{G}^{\prime}=\left(\mathrm{V}^{\prime}, \mathrm{E}^{\prime}\right)$ defined by $\left(\mu_{1}(a), \gamma_{1}(a)\right)=(0.3,0.4),\left(\mu_{1}(b), \gamma_{1}(b)\right)=(0.5,0.3),\left(\mu_{1}(c), \gamma_{1}(c)\right)=(0.6,0.4),\left(\mu_{1}(d), \gamma_{1}(d)\right)=(0.7,0.3)$, $\left(\mu_{2}(\mathrm{a}, \mathrm{b}), \gamma_{2}(\mathrm{a}, \mathrm{b})\right)=(0.1,0.2),\left(\mu_{2}(\mathrm{~b}, \mathrm{c}), \gamma_{2}(\mathrm{~b}, \mathrm{c})\right)=(0.3,0.2),\left(\mu_{2}(\mathrm{c}, \mathrm{d}), \gamma_{2}(\mathrm{c}, \mathrm{d})\right)=(0.5,0.3)$, $\left(\mu_{2}(\mathrm{~d}, \mathrm{a}), \gamma_{2}(\mathrm{~d}, \mathrm{a})\right)=(0.2,0.3) .\left(\mu_{1}\left(\mathrm{a}^{\prime}\right), \gamma_{1}\left(\mathrm{a}^{\prime}\right)\right)=(0.3,0.4),\left(\mu_{1}\left(\mathrm{~b}^{\prime}\right), \gamma_{1}\left(\mathrm{~b}^{\prime}\right)\right)=(0.5,0.3),\left(\mu_{1}\left(\mathrm{c}^{\prime}\right), \gamma_{1}\left(\mathrm{c}^{\prime}\right)\right)=(0.6,0.4)$, $\left(\mu_{1}\left(d^{\prime}\right), \gamma_{1}\left(d^{\prime}\right)\right)=(0.7,0.3),\left(\mu_{2}\left(a^{\prime}, b^{\prime}\right), \gamma_{2}\left(a^{\prime}, b^{\prime}\right)\right)=(0.2,0.1),\left(\mu_{2}\left(b^{\prime}, c^{\prime}\right), \gamma_{2}\left(b^{\prime}, c^{\prime}\right)\right)=(0.3,0.1)$, $\left(\mu_{2}\left(c^{\prime}, d^{\prime}\right), \gamma_{2}\left(c^{\prime}, d^{\prime}\right)\right)=(0.5,0.2),\left(\mu_{2}\left(d^{\prime}, a^{\prime}\right), \gamma_{2}\left(d^{\prime}, a^{\prime}\right)\right)=(0.2,0.1)$.

Then complement of $\mathrm{G}$ and $\mathrm{G}$ ' are $\bar{G}$ and $\bar{G}^{\prime}$ will be $\left(\left(\bar{\mu}_{1}(\mathrm{a}), \bar{\gamma}_{1}(\mathrm{a})\right)=(0.3,0.4),\left(\bar{\mu}_{1}(\mathrm{~b}), \bar{\gamma}_{1}(\mathrm{~b})\right)=(0.5,0.3)\right.$, $\left(\bar{\mu}_{1}(\mathrm{c}), \bar{\gamma}_{1}(\mathrm{c})\right)=(0.6,0.4),\left(\bar{\mu}_{1}(\mathrm{~d}), \bar{\gamma}_{1}(\mathrm{~d})\right)=(0.7,0.3),\left(\bar{\mu}_{2}(\mathrm{a}, \mathrm{b}), \bar{\gamma}_{2}(\mathrm{a}, \mathrm{b})\right)=(0.2,0.2),\left(\bar{\mu}_{2}(\mathrm{~b}, \mathrm{c}), \bar{\gamma}_{2}(\mathrm{~b}, \mathrm{c})\right)=(0.2,0.2)$, $\left(\bar{\mu}_{2}(\mathrm{c}, \mathrm{d}), \bar{\gamma}_{2}(\mathrm{c}, \mathrm{d})\right)=(0.1,0.1),\left(\bar{\mu}_{2}(\mathrm{~d}, \mathrm{a}), \bar{\gamma}_{2}(\mathrm{~d}, \mathrm{a})\right)=(0.1,0.1),\left(\bar{\mu}_{2}(\mathrm{a}, \mathrm{c}), \bar{\gamma}_{2}(\mathrm{a}, \mathrm{c})\right)=(0.3,0.4),\left(\bar{\mu}_{2}(\mathrm{~b}, \mathrm{~d}), \bar{\gamma}_{2}(\mathrm{~b}, \mathrm{~d})\right)=$ $(0.5,0.3)$. And $\left(\bar{\mu}_{1}\left(\mathrm{a}^{\prime}\right), \bar{\gamma}_{1}\left(\mathrm{a}^{\prime}\right)\right)=(0.3,0.4),\left(\bar{\mu}_{1}\left(\mathrm{~b}^{\prime}\right), \bar{\gamma}_{1}\left(\mathrm{~b}^{\prime}\right)\right)=(0.5,0.3),\left(\bar{\mu}_{1}\left(\mathrm{c}^{\prime}\right), \bar{\gamma}_{1}\left(\mathrm{c}^{\prime}\right)\right)=(0.6,0.4)$, $\left(\bar{\mu}_{1}\left(\mathrm{~d}^{\prime}\right), \bar{\gamma}_{1}\left(\mathrm{~d}^{\prime}\right)\right)=(0.7,0.3),\left(\bar{\mu}_{2}\left(\mathrm{a}^{\prime}, \mathrm{b}^{\prime}\right), \bar{\gamma}_{2}\left(\mathrm{a}^{\prime}, \mathrm{b}^{\prime}\right)\right)=(0.1,0.3),\left(\bar{\mu}_{2}\left(\mathrm{~b}^{\prime}, \mathrm{c}^{\prime}\right), \bar{\gamma}_{2}\left(\mathrm{~b}^{\prime}, \mathrm{c}^{\prime}\right)\right)=(0.2,0.3)$, $\left(\bar{\mu}_{2}\left(\mathrm{c}^{\prime}, \mathrm{d}^{\prime}\right), \bar{\gamma}_{2}\left(\mathrm{c}^{\prime}, \mathrm{d}^{\prime}\right)\right)=(0.1,0.2),\left(\bar{\mu}_{2}\left(\mathrm{~d}^{\prime}, \mathrm{a}^{\prime}\right), \bar{\gamma}_{2}\left(\mathrm{~d}^{\prime}, \mathrm{a}^{\prime}\right)\right)=(0.1,0.3),\left(\bar{\mu}_{2}\left(\mathrm{a}^{\prime}, \mathrm{c}^{\prime}\right), \bar{\gamma}_{2}\left(\mathrm{a}^{\prime}, \mathrm{c}^{\prime}\right)\right)=(0.3,0.4)$, $\left(\bar{\mu}_{2}\left(\mathrm{~b}^{\prime}, \mathrm{d}^{\prime}\right), \bar{\gamma}_{2}\left(\mathrm{~b}^{\prime}, \mathrm{d}^{\prime}\right)\right)=(0.5,0.3)$.

In this example, $\mathrm{G}$ is weak isomorphic with $\mathrm{G}^{\prime}$, and we get $\bar{G}^{\prime}$ is weak isomorphic with $\bar{G}$. But $\bar{G}$ is not neighbourly irregular IFG.

Theorem 4.6: Let $\mathrm{G}=(\mathrm{V}, \mathrm{E})$ be highly irregular and neighbourly Irregular Intutionistic fuzzy graph if and only if the degrees of all vertices of $\mathrm{G}$ are distinct.

Proof: Let $\mathrm{G}$ be a Intuitionistic fuzzy graph with $\mathrm{n}$ vertices $\mathrm{v}_{1}, \mathrm{v}_{2}, \ldots, \mathrm{v}_{\mathrm{n}}$ Assume $\mathrm{G}$ is highly Irregular IFG and neighbourly irregular IFG.

Let the adjacent vertices of $\mathrm{v}_{1}$ be $\mathrm{v}_{2}, \mathrm{v}_{3}, \ldots, \mathrm{v}_{\mathrm{n}}$ with degrees $\left(\mathrm{c}_{2}, \mathrm{k}_{2}\right),\left(\mathrm{c}_{3}, \mathrm{k}_{3}\right), \ldots,\left(\mathrm{c}_{\mathrm{n}}, \mathrm{k}_{\mathrm{n}}\right)$ respectively.

As $\mathrm{G}$ is highly irregular IFG, then either $\mathrm{c}_{2} \neq \mathrm{c}_{3} \neq, \ldots, \mathrm{c}_{\mathrm{n}}$ or $\mathrm{k}_{2} \neq \mathrm{k}_{3} \neq, \ldots, \mathrm{k}_{\mathrm{n}}$ or both

Therefore $\mathrm{d}\left(\mathrm{v}_{1}\right)$ cannot be $\left(\mathrm{c}_{2}, \mathrm{k}_{2}\right),\left(\mathrm{c}_{3}, \mathrm{k}_{3}\right), \ldots,\left(\mathrm{c}_{\mathrm{n}}, \mathrm{k}_{\mathrm{n}}\right)$ as $\mathrm{G}$ is neighbourly Irregular IFG.

i.e) the degrees of all vertices of $\mathrm{G}$ are distinct.

Conversely, assume that the degrees of all vertices of $G$ are distinct. That is every two adjacent vertices have distinct degrees and to every vertex the adjacent vertices have distinct degrees.

Hence $\mathrm{G}$ is neighbourly irregular IFG and highly irregular IFG.

\section{Isomorphic properties of Totally irregular IFG and its complement}

Definition 5.1 :Let $G=(V, E)$ be a IFG. Then G is totally irregular, if there is a vertex which is adjacent to vertices with distinct total degrees.

Definition 5.2: If every two adjacent vertices of a IFG,$G=(V, E)$ have distinct total degree, then $G$ is said to be a neighbourly total irregular IFG

\section{Proposition 5.3:}

Totally Irregular IFG need not be neighbourly total Irregular IFG.

\section{Example 5.4:}

$\left(\mu_{1}(\mathrm{a}), \gamma_{1}(\mathrm{a})\right)=(0.5,0.5),\left(\mu_{1}(\mathrm{~b}), \gamma_{1}(\mathrm{~b})\right)=(0.4,0.6),\left(\mu_{1}(\mathrm{c}), \gamma_{1}(\mathrm{c})\right)=(0.6,0.4),\left(\mu_{1}(\mathrm{~d}), \gamma_{1}(\mathrm{~d})\right)=(0.5,0.3)$

$\left(\mu_{2}(a, b), \gamma_{2}(a, b)\right)=(0.1,0.3),\left(\mu_{2}(b, c), \gamma_{2}(b, c)\right)=(0.2,0.3),\left(\mu_{2}(c, d), \gamma_{2}(c, d)\right)=(0.2,0.3)$,

$\left(\mu_{2}(d, a), \gamma_{2}(d, a)\right)=(0.3,0.4)$.

Here, $\mathrm{G}$ is totally Irregular But the adjacent vertices $\mathrm{c}$ and $\mathrm{d}$ have same total degree $(1,1), \mathrm{G}$ is not neighbourly total irregular IFG.

\section{Remark :}

The converse is not true. That is, every neighbourly Total irregular IFG is totally Irregular IFG except Intutionistic path graph.

Proposition 5.5: Let $G=(V, E)$ and $G^{\prime}=\left(V^{\prime}, E^{\prime}\right)$ be two neighbourly Total irregular intuitionistic fuzzy graphs, $\mathrm{G}$ and $\mathrm{G}$ ' are isomorphic if and only if their complements are isomorphic, but the complements need not be negihbourly total irregular IFG.

The proof is as same as Theorem 4.2. 
Example 5.6: Consider two neighbourly total irregular IFGs $G=(V, E)$ and $G^{\prime}=\left(V^{\prime}, E^{\prime}\right)$ defined by $\left(\mu_{1}(\mathrm{a}), \gamma_{1}(\mathrm{a})\right)=(0.2,0.3)=\left(\mu_{1}\left(\mathrm{a}^{\prime}\right), \gamma_{1}\left(\mathrm{a}^{\prime}\right),\left(\mu_{1}(\mathrm{~b}), \gamma_{1}(\mathrm{~b})\right)=(0.4,0.5)=\left(\mu_{1}\left(\mathrm{~b}^{\prime}\right), \gamma_{1}\left(\mathrm{~b}^{\prime}\right)\right),\left(\mu_{1}(\mathrm{c}), \gamma_{1}(\mathrm{c})\right)=(0.3,0.4)=\right.$ $\left(\mu_{1}\left(c^{\prime}\right), \gamma_{1}\left(c^{\prime}\right)\right),\left(\mu_{1}(d), \gamma_{1}(d)\right)=(0.3,0.5)=\left(\mu_{1}\left(d^{\prime}\right), \gamma_{1}\left(d^{\prime}\right)\right),\left(\mu_{2}(a, b), \gamma_{2}(a, b)\right)=(0.1,0.2)=\left(\mu_{2}\left(a^{\prime}, b^{\prime}\right), \gamma_{2}\left(a^{\prime}, b^{\prime}\right)\right)$, $\left(\mu_{2}(b, c), \gamma_{2}(b, c)\right)=(0.2,0.1)=\left(\mu_{2}\left(b^{\prime}, c^{\prime}\right), \gamma_{2}\left(b^{\prime}, c^{\prime}\right)\right),\left(\mu_{2}(c, d), \gamma_{2}(c, d)\right)=(0.2,0.4)=\left(\mu_{2}\left(c^{\prime}, d^{\prime}\right), \gamma_{2}\left(c^{\prime}, d^{\prime}\right)\right)$, $\left(\mu_{2}(\mathrm{~d}, \mathrm{a}), \gamma_{2}(\mathrm{~d}, \mathrm{a})\right)=(0.1,0.3)=\left(\mu_{2}\left(\mathrm{~d}^{\prime}, \mathrm{a}^{\prime}\right), \gamma_{2}\left(\mathrm{~d}^{\prime}, \mathrm{a}^{\prime}\right)\right),\left(\mu_{2}(\mathrm{~b}, \mathrm{~d}), \gamma_{2}(\mathrm{~b}, \mathrm{~d})\right)=(0.3,0.5)=\left(\mu_{2}\left(\mathrm{~b}^{\prime}, \mathrm{d}^{\prime}\right), \gamma_{2}\left(\mathrm{~b}^{\prime}, \mathrm{d}^{\prime}\right)\right)$. Then $\bar{G}$ and $\bar{G}^{\prime}$ will be $\left(\left(\bar{\mu}_{1}(\mathrm{a}), \bar{\gamma}_{1}(\mathrm{a})\right)=(0.2,0.3)=\left(\bar{\mu}_{1}\left(\mathrm{a}^{\prime}\right), \bar{\gamma}_{1}\left(\mathrm{a}^{\prime}\right)\right),\left(\bar{\mu}_{1}(\mathrm{~b}), \bar{\gamma}_{1}(\mathrm{~b})\right)=(0.4,0.5)=\left(\bar{\mu}_{1}\left(\mathrm{~b}^{\prime}\right), \bar{\gamma}_{1}\left(\mathrm{~b}^{\prime}\right)\right)\right.$, $\left(\bar{\mu}_{1}(\mathrm{c}), \bar{\gamma}_{1}(\mathrm{c})\right)=(0.3,0.4)=\left(\bar{\mu}_{1}\left(\mathrm{c}^{\prime}\right), \bar{\gamma}_{1}\left(\mathrm{c}^{\prime}\right)\right),\left(\bar{\mu}_{1}(\mathrm{~d}), \bar{\gamma}_{1}(\mathrm{~d})\right)=(0.3,0.5)=\left(\bar{\mu}_{1}\left(\mathrm{~d}^{\prime}\right), \bar{\gamma}_{1}\left(\mathrm{~d}^{\prime}\right)\right),\left(\bar{\mu}_{2}(\mathrm{a}, \mathrm{b}), \bar{\gamma}_{2}(\mathrm{a}, \mathrm{b})\right)=(0.1,0.3)=$ $\left(\bar{\mu}_{2}\left(\mathrm{a}^{\prime}, \mathrm{b}^{\prime}\right), \bar{\gamma}_{2}\left(\mathrm{a}^{\prime}, \mathrm{b}^{\prime}\right)\right),\left(\bar{\mu}_{2}(\mathrm{~b}, \mathrm{c}), \bar{\gamma}_{2}(\mathrm{~b}, \mathrm{c})\right)=(0.1,0.4)=\left(\bar{\mu}_{2}\left(\mathrm{~b}^{\prime}, \mathrm{c}^{\prime}\right), \bar{\gamma}_{2}\left(\mathrm{~b}^{\prime}, \mathrm{c} \mathrm{c}^{\prime}\right)\right),\left(\bar{\mu}_{2}(\mathrm{c}, \mathrm{d}), \bar{\gamma}_{2}(\mathrm{c}, \mathrm{d})\right)=(0.1,0.1)=$ $\left(\bar{\mu}_{2}\left(\mathrm{c}^{\prime}, \mathrm{d}^{\prime}\right), \bar{\gamma}_{2}\left(\mathrm{c}^{\prime}, \mathrm{d}^{\prime}\right)\right),\left(\bar{\mu}_{2}(\mathrm{~d}, \mathrm{a}), \bar{\gamma}_{2}(\mathrm{~d}, \mathrm{a})\right)=(0.1,0.3)=\left(\bar{\mu}_{2}\left(\mathrm{~d}^{\prime}, \mathrm{a}^{\prime}\right), \bar{\gamma}_{2}\left(\mathrm{~d}^{\prime}, \mathrm{a}^{\prime}\right)\right)$,

$\left(\bar{\mu}_{2}(\mathrm{a}, \mathrm{c}), \bar{\gamma}_{2}(\mathrm{a}, \mathrm{c})\right)=(0.2,0.4)=\left(\bar{\mu}_{2}\left(\mathrm{a}^{\prime}, \mathrm{c}^{\prime}\right), \bar{\gamma}_{2}\left(\mathrm{a}^{\prime}, \mathrm{c}^{\prime}\right)\right)$.

Here $\mathrm{G}$ is isomorphic with $\mathrm{G}^{\prime}$, and $\bar{G}$ is also isomorphic with $\bar{G}^{\prime}$. But the complements are not neighbourly total irregular IFGs.

Theorem 5.7: Let $\mathrm{G}=(\mathrm{V}, \mathrm{E})$ be neighbourly total Irregular intuitionistic fuzzy graph and if $\mu_{2}(\mathrm{x}, \mathrm{y}) \leq 1 / 2 \mu_{1}(x) \Lambda \mu_{1}(y)$ and $\gamma_{2}(\mathrm{x}, \mathrm{y}) \geq 1 / 2 \gamma_{1}(\mathrm{x}) \mathrm{V} \gamma_{1}(\mathrm{y})$ for every $\mathrm{x}, \mathrm{y} \in \mathrm{V}$, then $\mathrm{G}$ is self weak complementary IFG only. But the complement need not be neighbourly total irregular Intutitonistic fuzzy graph.

Proof: Let $\mathrm{G}=(\mathrm{V}, \mathrm{E})$ be neighbourly total irregular IFG with

$\mu_{2}(\mathrm{x}, \mathrm{y}) \leq 1 / 2 \mu_{1}(x) \Lambda \mu_{1}(y)$ and $\gamma_{2}(\mathrm{x}, \mathrm{y}) \geq 1 / 2 \gamma_{1}(\mathrm{x}) \mathrm{V} \gamma_{1}(\mathrm{y})$.

Consider the identity map h: $\mathrm{S} \rightarrow \mathrm{S}$ such that $\left(\mu_{1}(\mathrm{x}), \gamma_{1}(\mathrm{x})\right)=\left(\bar{\mu}_{1}(\mathrm{x}, \mathrm{y}), \bar{\gamma}_{l}(\mathrm{x}, \mathrm{y})\right.$ for every $\mathrm{x} C \mathrm{~S}$

By the definition of the $\mu$ complement,

$\bar{\mu}_{2}(\mathrm{x}, \mathrm{y})=\left(\mu_{1}(\mathrm{x}) \Lambda \mu_{1}(\mathrm{y})-\mu_{2}(\mathrm{x}, \mathrm{y}) \geq\left(\mu_{1}(\mathrm{x}) \Lambda \mu_{1}(\mathrm{y})-1 / 2\left(\mu_{1}(\mathrm{x}) \Lambda \mu_{1}(\mathrm{y})\right)=1 / 2\left(\mu_{1}(\mathrm{x}) \Lambda \mu_{1}(\mathrm{y})\right)\right.\right.$

$\bar{\mu}_{2}(\mathrm{x}, \mathrm{y}) \geq \mu_{2}(\mathrm{x}, \mathrm{y}) \rightarrow \mu_{2}(\mathrm{x}, \mathrm{y}) \leq \bar{\mu}_{2}(\mathrm{x}, \mathrm{y})$ for every $\mathrm{x}, \mathrm{y} \in \mathrm{S}$

Similarly, by the definition of $\gamma$ complement,

$\bar{\gamma}_{2}(\mathrm{x}, \mathrm{y})=\gamma_{1}(\mathrm{x}) \mathrm{V} \gamma_{1}(\mathrm{y})-\gamma_{2}(\mathrm{x}, \mathrm{y}) \leq \gamma_{1}(\mathrm{x}) \mathrm{V} \gamma_{1}(\mathrm{y})-1 / 2\left(\gamma_{1}(\mathrm{x}) \mathrm{V} \gamma_{1}(\mathrm{y})\right)=1 / 2\left(\gamma_{1}(\mathrm{x}) \mathrm{V} \gamma_{1}(\mathrm{y})\right)$

$\bar{\gamma}_{2}(\mathrm{x}, \mathrm{y}) \leq \gamma_{2}(\mathrm{x}, \mathrm{y}) \rightarrow \gamma_{2}(\mathrm{x}, \mathrm{y}) \geq \bar{\gamma}_{2}(\mathrm{x}, \mathrm{y})$ for every $\mathrm{x}, \mathrm{y} \in \mathrm{S}$

i.e) $\mathrm{G}$ is self weak complementary IFG. But the complement need not be neighbourly total irregular IFG.

\section{Example 5.8:}

$\left(\mu_{1}(\mathrm{a}), \gamma_{1}(\mathrm{a})\right)=(0.5,0.4),\left(\mu_{1}(\mathrm{~b}), \gamma_{1}(\mathrm{~b})\right)=(0.4,0.6),\left(\mu_{1}(\mathrm{c}), \gamma_{1}(\mathrm{c})\right)=(0.7,0.3),\left(\mu_{1}(\mathrm{~d}), \gamma_{1}(\mathrm{~d})\right)=(0.8,0.2)$,

$\left(\mu_{2}(a, b), \gamma_{2}(a, b)\right)=(0.1,0.4),\left(\mu_{2}(b, c), \gamma_{2}(b, c)\right)=(0.1,0.3),\left(\mu_{2}(c, d), \gamma_{2}(c, d)\right)=(0.3,0.2),\left(\mu_{2}(d, a), \gamma_{2}(d, a)\right)=(0.2,0.2)$.

Then $\bar{G}$ is defined as $\left(\left(\bar{\mu}_{1}(\mathrm{a}), \bar{\gamma}_{1}(\mathrm{a})\right)=(0.5,0.4),\left(\bar{\mu}_{1}(\mathrm{~b}), \bar{\gamma}_{1}(\mathrm{~b})\right)=(0.4,0.6),\left(\bar{\mu}_{1}(\mathrm{c}), \bar{\gamma}_{1}(\mathrm{c})\right)=(0.7,0.3)\right.$,

$\left(\bar{\mu}_{1}(\mathrm{~d}), \bar{\gamma}_{1}(\mathrm{~d})\right)=(0.8,0.2),\left(\bar{\mu}_{2}(\mathrm{a}, \mathrm{b}), \bar{\gamma}_{2}(\mathrm{a}, \mathrm{b})\right)=(0.3,0.2),\left(\bar{\mu}_{2}(\mathrm{~b}, \mathrm{c}), \bar{\gamma}_{2}(\mathrm{~b}, \mathrm{c})\right)=(0.3,0.3),\left(\bar{\mu}_{2}(\mathrm{c}, \mathrm{d}), \bar{\gamma}_{2}(\mathrm{c}, \mathrm{d})\right)=(0.4,0.1)$, $\left(\bar{\mu}_{2}(\mathrm{~d}, \mathrm{a}), \bar{\gamma}_{2}(\mathrm{~d}, \mathrm{a})\right)=(0.3,0.2),\left(\bar{\mu}_{2}(\mathrm{a}, \mathrm{c}), \bar{\gamma}_{2}(\mathrm{a}, \mathrm{c})\right)=(0.5,0.4),\left(\bar{\mu}_{2}(\mathrm{~b}, \mathrm{~d}), \bar{\gamma}_{2}(\mathrm{~b}, \mathrm{~d})\right)=(0.4,0.6)$.

In the above example $\mathrm{G}$ and $\bar{G}$ are self weak isomorphic, $\mathrm{G}$ is neighbourly total irregular IFG but $\bar{G}$ is not neighbourly total irregularIFG. So neighbourly total irregular IFG need not be self weak complementary neighbourly total irregular IFG.

\section{Conclusion}

Here we derived and discussed some more Isomorphism properties of highly Irregular Intuitionistc Fuzzy Graph and isomorphism properties of neighbourly Irregular Intuitionistc Fuzzy Graphs and its complement are discussed. Finally, we studied Isomorphism on neigbourly total Irregular IFG and its complements

\section{References}

[1] Atanassov. KT. Intuitionistic fuzzy sets: theory and applications. Physica, New York, 1999.

[2] Parvathi, R. and Karunambigai, M.G., Intuitionistic Fuzzy Graphs, Computational Intelligence, Theory and applications, International Conference in Germany, Sept $18-20,2006$.

[3] A.NagoorGani and S.R. Latha, On Irregular Fuzzy Graphs, Applied Mathematical Sciences,Vol.6, 2012, no.11,517-523.

[4] A.NagoorGani and S.Shajitha Begum, Degree,Order and Size in Intuitionistic Fuzzy Graphs,International Journal of Algorithms, Computing and Mathematics,(3)3 (2010).

[5] Bhutani,K.R., On Automorphism of Fuzzy graphs, Pattern Recognition Letter 9:159-162,1989.

[6] NagoorGani, A. and Malarvizhi. J, Isomorphism on Fuzzy graphs, International Journal of Computational and Mathematical Sciences 2;4:200-206,2008.

[7] Sunitha, M.S. and Vijayakumar, A., Complement of a Fuzzy graph, Indian J. Pure appl. Math. 33(9):1451-1464, September 2002.

[8] Harary,F., Graph Theory, Addition Wesley, Third Printing, October 1972.

[9] Zimmermann, H.J., Fuzzy Set Theory and its Applications, Kluwer-Nijhoff, Boston, 1985.

[10] NagoorGani.A et. al ,Irregular Intuitionistic fuzzy graph, IOSR Journal Mathematics Vol. 9, Issue 6 (Jan. 2014$)$, PP 47-51.

[11] JahirHussain. R and YahyaMohamed.S , Properties on Irregular Intuitionistic Fuzzy Graphs(IIFG), Applied Mathematical Sciences, Hikari Ltd, Bulgaria, Vol. 8, 2014 , no.8, pp.379-389. 